Interessen zunehmend verschwimmen würden. Der Autor spricht dabei von einem Ende der Öffentlichkeit, wenn öffentliche Institutionen (wie die Medien selbst) ihre Unabhängigkeit verlieren würden und auf den politischen Bereich angewiesen bzw. von diesem abhängig wären. Die Presse, ursprünglich auf dem Räsonnement des Publikums basierend und als Art verlängerter Arm der Bürger_ innen fungierend, entwickele sich im Zuge der Kommerzialisierung zu einer Verlängerung politischer und wirtschaftlicher Interessen. Insofern könne die Presse auch ihre kritische Funktion zunehmend verlieren.

Habermas bezieht sich in seiner Schrift «Strukturwandel der Öffentlichkeit» (2018 [1962]) auf die Entwicklung der Öffentlichkeit in den vergangenen Jahrhunderten bis hin zum 20. Jahrhundert. Dabei handelt es sich um detaillierte theoretische und historische Beobachtungen des Mediensystems, die mit Blick auf aktuelle Entwicklungen im 21. Jahrhundert noch immer in hohem Masse griffig sind. Dennoch brachten der digitale Wandel und die Etablierung neuer Medien erneut die Frage hervor, inwiefern sich die öffentliche Kommunikation weiterentwickelt. In diesem Kontext wird in aktueller, vor allem theoretischer Forschung häufig von einer digitalen Transformation oder einem digitalen Strukturwandel der Öffentlichkeit gesprochen (z. B. Eisenegger, 2017; Fraser, 2010; Hagen, Wieland \& In der Au, 2017; Helmond, 2015; Thiel, 2016). Diese Annahmen basieren in erster Linie auf der Annahme einer Veränderung des öffentlichen Raumes aufgrund neuer Möglichkeiten der öffentlichen Kommunikation über das Internet oder über Social-Media-Plattformen. Für die vorliegende Arbeit ist diesbezüglich von einer zentralen theoretischen Überlegung zu sprechen, die eine Neudeutung des Öffentlichkeitsund Gegenöffentlichkeitsbegriffs in Frage stellt. Das folgende Kapitel gibt daher einen Überblick über aktuelle theoretische Überlegungen zum Öffentlichkeitsbegriff, wobei vor allem auf die Veränderung des Mediensystems respektive der Öffentlichkeit basierend auf dem digitalen Wandel eingegangen wird.

\title{
II.3 Die digitale Transformation der Öffentlichkeit
}

Habermas' Ausführungen zum Strukturwandel der Öffentlichkeit laden dazu ein, diese für das 21. Jahrhundert und allenfalls zukünftige Entwicklungen weiterzudenken. In aktuellen theoretischen Arbeiten wird in diesem Zusammenhang oftmals von einem dritten Strukturwandel der Öffentlichkeit oder einer digitalen Transformation der Öffentlichkeit gesprochen (z. B. Eisenegger, 2017; Fraser, 2010; Hagen et al., 2017; Helmond, 2015; Thiel, 2016). Der erste und der zweite Strukturwandel lassen sich von Habermas (2018 [1962]), Münch (1995) oder Imhof (2000) ableiten (vgl. hierzu auch Eisenegger, 2021). Zusammenfassend lässt sich der erste Strukturwandel der Öffentlichkeit im 18. und 19. Jahrhundert verorten, als durch das Leitmedium der Zeitung erstmalig Öffentlichkeit massenmedial hergestellt 
wurde, die sich vormals insbesondere im Rahmen von Debattierklubs, Salons oder Kaffeehäuser konstituierte. Es handelte sich hierbei um Partei- und Gesinnungspresse, die entsprechend Positionen der jeweiligen Trägerorganisation vermittelte, seien es politische Parteien, religiöse Organisationen (Kirchen) u. ä., die bis ins 20. Jahrhundert öffentlich wirkmächtig waren. Der zweite Strukturwandel schaffte einen Abbruch dieser meinungsorientierten, politisch-ideologischen Gesinnungspresse; das Mediensystem wandelte sich nach ökonomischen Prämissen und unterlag einer Kommerzialisierung: Massgeblich wurden die Publikumsinteressen, private Medienkonzerne etablierten sich und konzentrierten sich nicht nur auf die Publikation von Informationen, sondern vor allem auch von Unterhaltungsbeiträgen, beispielsweise in Form von Boulevard- oder Special-Interest-Medien, und das Fernsehen entwickelte sich zum zentralen Leitmedium. Die Bereitschaft, für Nachrichten zu zahlen, war in dieser Zeit noch hoch (Eisenegger, 2017, 2021).

Aktuell stellt sich die Frage nach einem dritten oder digitalen Strukturwandel (oder auch einer digitalen Transformation) der Öffentlichkeit. Aktuelle Öffentlichkeitskonzepte streichen dabei vor allem den Stellenwert von Digitalplattformen heraus. Einige Ansätze, die aktuelle theoretische Überlegungen repräsentieren, sollen an dieser Stelle erwähnt werden. Vorweggenommen werden muss jedoch, dass sich auch die Begrifflichkeit von «Öffentlichkeit» gewandelt hat respektive von verschiedenen Autor_innen in unterschiedlicher Weise gefasst wird. Diese sollen folgend - ohne Anspruch auf Vollständigkeit - skizziert werden.

\section{II.3.1 Netzwerkgesellschaft und -öffentlichkeit}

Basierend auf dem Netzwerkcharakter des Internets sind in diesem Kontext vor allem Konzepte der «Netzwerköffentlichkeit» oder «Netzwerkgesellschaft» hervorzuheben. Castells (2007, 2010 [1996]) ist für den Kontext dieser Arbeit als wesentlich herauszustreichen. In seiner Theorie zur Netzwerkgesellschaft beschäftigt sich Castells (2010 [1996]) schon vor der Wende zum 21. Jahrhundert mit der Transformation der Gesellschaft hin zu einer globalen Verknotung von Individuen, basierend auf Macht, Kapital, Information respektive Kommunikation und technisch basierten Logiken. Es handelt sich auch hierbei um keinen empirisch basierten, netzwerkanalytischen Ansatz. Zentral in Castells Theorie ist die Annahme einer zunehmend globaler werdenden Netzwerkökonomie, die über Finanzströme getrieben wird. Zudem betont der Autor eine dahingehende Rolle von Macht und damit einhergehenden Ungleichheiten, die beispielsweise durch soziale Bewegungen thematisiert werden. Weiter sieht Castells als wesentliches Kriterium der Netzwerkgesellschaft den virtuellen Raum, indem Kommunikation zwischen Individuen ort- und zeitunabhängig stattfindet - im Sinne einer Vernetzung von Akteur_innen. In diesem Kontext spielen sowohl Individualisie- 
rungs- als auch Fragmentierungsprozesse eine Rolle, die von einer Suche nach Identität geprägt sind. Dies kann sich in Form sozialer Protestbewegungen oder zivilgesellschaftlichem Engagement äussern - beispielsweise via Social Media (von Kardoff, 2019).

In Castells (2007) Aufsatz «Communication, Power and Counter-power in the Network Society» beschäftigt sich der Autor konkret mit den Relationen zwischen Kommunikation und Macht. Castells sieht Medien als jenen Raum, in dem Macht auf Basis von Information und Kommunikation ausgehandelt wird, und bezieht sich dabei vor allem auf die dahingehende Rolle des Internets. Macht definiert Castells (2007, S. 239) als die Kompetenz eines Akteurs/einer Akteurin, den eigenen Willen gegenüber anderen sozialen Akteur_innen durchzusetzen - ähnlich der Weberschen (1984 [1921], S. 89) Definition: «Macht bedeutet jede Chance, innerhalb einer sozialen Beziehung den eigenen Willen auch gegen Widerstreben durchzusetzen, gleichviel worauf diese Chance beruht». In diesem Zusammenhang ist auch der Begriff der Gegenmacht (counter-power) wesentlich, definiert als die Kompetenz von Akteur_innen, sich institutionalisierten Machtbeziehungen zu widersetzen (Castells, 2007, S. 239). Gesellschaftlichen Wandel sieht Castells vor allem in den Dynamiken dieser Machtbeziehungen begründet. Eine wesentliche Rolle nehmen in diesem Zusammenhang die Massenmedien, als Raum für die Aushandlung von Macht, und deren Transformation im Internetzeitalter ein. Während die Massenmedien von einer One-way-Kommunikation geprägt sind, basiert die Netzwerkgesellschaft auf interaktiver, ort- und zeitunabhängiger Kommunikation. Durch die digitale Transformation haben Individuen zudem ihr eigenes System der (Massen-)Kommunikation entwickelt, nämlich über mobile Kommunikation, Blogs, Wikis und insgesamt Social-Media-Plattformen. Castells (2007, S. 246) subsumiert diese Entwicklung unter dem Begriff «mass self-communication», worunter die Verbreitung von selbstgeneriertem Inhalt für ein globales Publikum gefasst wird. Im Kontext der Gegenmacht fungiert diese mass self-communication als Möglichkeit, institutionalisierte Machtverhältnisse $\mathrm{zu}$ transformieren oder zumindest $\mathrm{zu}$ hinterfragen, beispielsweise in Form von sozialen Bewegungen. Gleichzeitig nutzen auch politische Eliten die mass self-communication im digitalen Zeitalter, um mit ihrem Publikum in Diskurs zu treten. Castells (2007) streicht zudem die Koexistenz von Massenmedien und neuen Medien heraus, die nicht in Widerspruch oder Konkurrenz zueinanderstehen, sondern auch unterstützend agieren können, beispielsweise indem Digitalplattformen die Nachrichtenbeiträge von Massenmedien weiter streuen.

Auch weitere Autor_innen heben den Netzwerkcharakter der Gesellschaft respektive öffentlicher Kommunikation hervor. So sprechen beispielsweise Reese und Shoemaker (2016) von einer «Networked Public Sphere», einer vernetzten Öffentlichkeit. Die Autor_innen beziehen sich dabei vor allem auf die Veränderung des Journalismus durch die Digitalisierung, der nicht nur mit ökonomischer 
Ressourcenminimierung zu kämpfen hat, sondern auch aufgrund von freiverfügbaren Online-Inhalten (die eben nicht nur von professionell journalistischen Akteur_innen erstellt werden) seine Gatekeeping-Funktion, im Sinne der Auswahl, Verarbeitung und Distribution von Nachrichtenbeiträgen, zunehmend verliert (vgl. hierzu auch Wallace, 2017). Reese und Shoemaker (2016) fokussieren in diesem Zusammenhang mit ihrem «Hierarchy of Influences»-Modell unterschiedliche Ebenen, die Medieninhalte beeinflussen. Diese Ebenen beziehen sich sowohl auf Einflüsse der Makro-, Meso- als auch Mikrobene, darunter soziale Systeme, soziale Institutionen, Medienorganisationen, journalistische Routinen und schliesslich die Ebene der Individuen. Auch Friedland, Hove und Rojas (2014) arbeiten mit dem Begriff der «Networked Public Sphere» und beziehen sich dabei auf Habermas' Öffentlichkeitstheorie (2018 [1962]), unter der Annahme, dass es einer Revision der Theorie mit Blick auf die Netzwerkgesellschaft bedürfe. Die Autoren gehen in diesem Zusammenhang erstens davon aus, dass die kommunikative Sozialisation, die Habermas (2009 [1981]) in seiner «Theorie des kommunikativen Handelns» vor allem durch Familie und Institutionen wie die der Schule geprägt sieht, durch die Mediensozialisation ergänzt werden müsse, um einen rationalen Diskurs führen zu können. Zweitens nehmen Friedland et al. (2014) an, dass das Modell öffentlicher Kommunikation in Netzwerkumgebungen nicht mehr halte; so steige beispielsweise die Reflexivität des Diskurses durch entstehende Online-Öffentlichkeiten, die im Online-Diskurs argumentieren, diskutieren und politische Eliten kritisieren könnten. Drittens prophezeien die Autoren (ähnlich wie Castells (2010 [1996]) komplexer werdende Kommunikationsflüsse in der Netzwerköffentlichkeit. Auch Benkler (2006) versteht unter «Networked Public Sphere» die Praktiken, Organisationen und Technologien vernetzter Kommunikation, die eine alternative Arena für öffentlichen Diskurs, Interaktion und Mobilisierung ermöglichen (Benkler, Roberts, Faris, Solow-Niederman \& Etling, 2015, S. 596; vgl. hierzu auch Keller, 2019).

Nicht verwunderlich beziehen sich auch aktuelle Arbeiten, die sich mit der empirischen Analyse von Social-Media-Netzwerken beschäftigen, auf den Begriff der «Networked Public Sphere». Beispielhaft können in diesem Zusammenhang Arbeiten von Ausserhofer und Maireder (2013; Maireder \& Schlögl, 2016) genannt werden, die sich mit der österreichischen Twittersphäre netzwerkanalytisch auseinandersetzen, oder auch von Bruns und Kollegen (Bruns, Burgess, Highfield, Kirchhoff \& Nicolai, 2010; Bruns \& Stieglitz, 2012, 2013), die soziale Netzwerke auf Digitalplattformen in Australien analysieren. Es ist naheliegend, dass sich vor allem durch den Untersuchungsgegenstand von Digitalplattformen, die auf Netzwerklogiken basieren, der Öffentlichkeitsbegriff selbst entsprechend transformiert hat. In ähnlicher Weise werden in weiteren aktuellen Studien und theoretischen Arbeiten ebendiese Plattformen selbst als tragend für den Öffentlich- 
keitswandel betrachtet. Im folgenden Kapitel soll daher näher auf den Begriff der «Plattformöffentlichkeit» eingegangen werden.

\section{II.3.2 Plattformöffentlichkeit und Plattformisierung}

Die Etablierung von Digitalplattformen wird in vielen aktuellen Arbeiten als massgeblich für den Öffentlichkeitswandel betrachtet. In diesem Zusammenhang wird häufig - bezugnehmend auf Habermas' (2018 [1962]) Schrift zum Strukturwandel der Öffentlichkeit - von einem weiteren Strukturwandel gesprochen. Die genaue Begrifflichkeit variiert - so sprechen beispielsweise Hagen, Wieland und In der Aue (2017) von einem algorithmischen Strukturwandel. Die Autor_innen gehen davon aus, dass der durch die Massenmedien definierte Strukturwandel durch die Logik von Algorithmen abgelöst wurde, wonach Informationsintermediäre (im Sinne von Plattformen) eine neue Gatekeeper-Funktion der Medien einnehmen. Als problematisch erachten sie dabei die Tatsache, dass durch personalisierte Algorithmen eine Verzerrung, Fragmentierung und Polarisierung der Öffentlichkeit hervorgerufen wird (vgl. Kapitel II.3.3). Häufig fällt im Zusammenhang mit dem digitalen Wandel der Öffentlichkeit auch der Plattform-Begriff. Helmond (2015) beispielsweise spricht von einer «Platformization» respektive «Plattformisierung». Darunter fasst sie vor allem das ökonomische Modell von Plattformen und deren Ausweitung, die wesentlich für das Social Web und schliesslich die öffentliche Kommunikation sind. Helmond (2015) geht von einer dualen Logik der Plattformisierung aus, wonach Plattformen einerseits eine Infrastruktur für andere Websites und Apps schaffen, andererseits externe Daten (so beispielsweise die Beiträge von Newssites) für das eigene ökonomische Modell verwendet werden. Ebenso aus einer ökonomischen Perspektive argumentieren Kirchner und Beyer (2016), indem sie davon ausgehen, dass ökonomische Aktivitäten zunehmend auf digitale Märkte respektive Plattformen verlegt werden, was kennzeichnet für den Strukturwandel ist. Sie betrachten digitale Marktordnungen als «Plattformlogik».

Auch Eisenegger (2017) zufolge zeichnet sich der dritte oder digitale Strukturwandel der Öffentlichkeit seit den frühen 200oer Jahren ab. Geprägt ist dieser Öffentlichkeitswandel nicht nur durch die Digitalisierung, sondern im Speziellen - so Eisenegger (2021) - auch durch eine Plattformisierung. Darunter ist ein Aufschwung von globalen Tech-Plattformen, wie Google, Facebook, Twitter und Co., zu verstehen, die eine Funktion als Intermediäre einnehmen und massgeblich «auf die Struktur und die Inhalte öffentlicher Kommunikation» einwirken (Eisenegger, 2021, S. 18). Das Internet und soziale Netzwerke in Form von Digitalplattformen werden dabei als zentrale Instanz moderner Öffentlichkeit betrachtet, wobei vor allem mit der Entwicklung hin zu einer «Longtail»-Öffentlichkeit argumentiert wird (Anderson, 2006; Eisenegger, 2017; Neuberger, 2009, 2011). Der 
von Chris Anderson (2006) massgeblich geprägte «Longtail»-Begriff bezieht sich ursprünglich auf den Erfolg von Online-Verkaufsplattformen, wie z. B. Amazon und Ebay, und deren breites Angebot an Nischenprodukten. Diese sind im Gegensatz zu Bestsellern am «langen Schweif» der Umsatzkurve einzuordnen, wodurch unterschiedlichste Zielgruppen erreicht werden können. Die Longtail-Öffentlichkeit repräsentiert analog dazu ein Kontinuum an verschiedenen öffentlich agierenden Akteur_innen, die über unterschiedliche Reichweiten verfügen. Dieses Öffentlichkeitsbild grenzt sich z. B. von Gerhards' und Neidhardts (1991) Drei-Ebenen-Modell $\mathrm{ab}$, da nicht mehr von getrennt voneinander zu betrachtenden Öffentlichkeitsebenen ausgegangen wird. Die Longtail-Öffentlichkeit kann in diesem Zusammenhang als Weiterentwicklung dieses Ebenenmodells betrachtet werden, die notwendigerweise aufgrund der digitalen Transformation der Öffentlichkeit erfolgen muss. Diese Longtail-Öffentlichkeit ist entsprechend dadurch gekennzeichnet, dass im vorderen Teil eine zunehmende Medienkonzentration reichweitenstarker Medien festzustellen ist, während am «langen Schweif» - aufgrund ökonomischer und sozialer Einflüsse und insbesondere durch den Einfluss globaler Tech-Intermediäre - das Angebot reichweitenschwacher Informationsangebote zunimmt (Eisenegger, 2017, 2021). Die «ökonomische Plattformisierung» (Eisenegger, 2017, S. 15) bezieht sich auf traditionelle Kommunikator_ innen der Massenmedien, die in diesem Zusammenhang aus ökonomischer Sicht mit den Digitalplattformen konkurrieren. Diese Plattformen (vor allem Google und Facebook) beziehen mittlerweile einen Grossteil der Werbegelder ${ }^{1}$ und häufen gleichbedeutend ihre Marktmacht im Online-Bereich. Grund hierfür ist das von den Plattformen praktizierte Data Driven Advertising und User Targeting (Eisenegger, 2021; Lobigs, 2017). Dabei handelt es sich um personalisierte Werbung, die auf den persönlichen Daten der Nutzenden beruht und somit zielgruppen- bzw. personengerecht eingesetzt wird. Folgen für den professionellen Journalismus sind im Sinne einer Deinstitutionalisierung unter anderem eine Konzentration journalistischer Medienangebote sowie reduzierte Personalressourcen, verbunden mit einem Personalabbau (Eisenegger, 2021). Auch Hindman (2009) geht von einem Winner-Takes-All-Prinzip in Bezug auf die Longtail-Öffentlichkeit aus, indem er beschreibt, dass vor allem Digitalplattformen wie Google die Gewinner des Longtails sind. In Summe sind es nur wenige Websites, die Traffic erzielen und eben nicht kleinere, reichweitenschwache Websites, die zusammengenommen an Reichweite gewinnen.

Die «soziale Plattformisierung» beschreibt das Phänomen eines «entbündelten Medienkonsums» (Eisenegger, 2021, S. 32), wobei «Entbündelung» in diesem Kontext bedeutet, dass Medienmarken nicht mehr direkt von den Nutzer_innen ange-

1 In der Schweiz beispielsweise sammelte Google im Jahr 2018 mehr Werbeeinnahmen als die gesamte Schweizer Presse (Schwaiger, 2019). 
steuert (beispielsweise über das Aufrufen der Medien-Websites oder auch die Suche konkreter Medienmarken über Online-Suchmaschinen wie Google), sondern «emergent» auf Plattformen rezipiert werden, $d$. h. Inhalte werden basierend auf Algorithmen und persönlichen Daten den Rezipierenden zugespielt, beispielsweise über News-Aggregatoren oder die Newsfeeds auf Social-Media-Plattformen (Eisenegger, 2021, S. 32; Schmidt, Hölig, Merten \& Hasebrink, 2017; Schwaiger, 2019; Schwaiger, Schneider \& Vogler, 2020). Dies ist für den professionellen Informationsjournalismus insofern problematisch, als dieser Prozess in einer sukzessiven Deinstitutionalisierung traditioneller Informationsmedien mündet. Die Markenbindung von Nutzer_innen geht durch diese «Medienemergenz» verloren, wonach den Rezipierenden nicht mehr bewusst ist, welche Angebote sie tatsächlich nutzen (Eisenegger, 2021, S. 32) ${ }^{2}$.

Wesentlich ist laut Eisenegger (2017, 2021) die sogenannte «Plattformisierung», wie sie auch von Helmond (2015) beschrieben wird, die die digitale Transformation der Öffentlichkeit massgeblich prägt. Der Begriff umschreibt die Etablierung von Digitalplattformen in der Öffentlichkeit, d. h. den Bedeutungszuwachs dieser sowohl in räumlicher (Internet) als auch sozialer Hinsicht, wonach Plattformen gesellschaftliche Steuerungsprozesse übernehmen: «Sie kontrollieren die zentralen Zugänge zum Internet, strukturieren und überwachen die Bewegungsmöglichkeiten der Nutzerinnen und Nutzer, sie kuratieren, kontrollieren und selektionieren in großem Stil Inhalte, Informationsflüsse und Diskussionen» (Eisenegger, 2021, S. 22). Plattformen repräsentieren, dieser These folgend, ein Machtmonopol der Öffentlichkeit, das vor allem auf Datafizierung und Algorithmisierung basiert. Zudem finden auch im professionell journalistischen Bereich Anpassungen an diese Logiken statt, wonach traditionelle Medienangebote der gesellschaftlichen Erwartung einer Präsenz auf Digitalplattformen gerecht werden. ${ }^{3}$

2 In diesem Zusammenhang ist auch die Zahlungsbereitschaft der Nutzenden für einzelne Medienmarken in Frage zu stellen, da auf Digitalplattformen einzelne Marken eine geringere Rolle einnehmen als das Cesamtangebot unterschiedlicher Angebote, die ein personifiziertes, gebündeltes Medienmenü erstellen.

3 Eisenegger (2021) spricht von koerzivem, mimetischem und normativem Druck an die professionell journalistischen Medienangebote und bezieht sich dabei auf die Theorie des soziologischen Neo-Institutionalismus, wonach Organisationen gesellschaftliche Erwartungen erfüllen müssen, um als legitim zu gelten. Koerziver Anpassungsdruck bezieht sich in diesem Kontext auf zwanghaften Druck ausgehend von Digitalplattformen, dass journalistische Organisationen in Suchmaschinen und sozialen Plattformen überhaupt aufscheinen. Mimetischer Druck bezieht sich auf die Imitation von Digital plattformen (z. B. durch Einführung von metrischen Indikatoren wie Likes und Shares auf den eigenen Plattformen). Normativer Druck zeigt sich in der Anpassung der Organisationen an Professionalisierungsprozesse (z. B. Datenjournalismus, automatisierter Journalismus). 
Wie bereits angedeutet, impliziert die Etablierung von Plattformen auch eine (potentielle) Entwicklung von «Plattformlogiken» (Eisenegger, 2021), «Social Media Logics» (van Dijck \& Poell, 2013) oder auch «Network Media Logics» (Klinger \& Svensson, 2015). Van Dijk und Poel (2013) erachten dabei die Vernetzung als eigene Social Media Logic neben der Programmierbarkeit der Kommunikation im Sinne einer Algorithmisierung, Popularität durch Likes und der Datafikation. Klinger und Svensson (2015) sprechen wiederum von einer Network Media Logic, die vor allem dadurch geprägt sei, dass es hinsichtlich Produktion, Distribution und Mediennutzung Unterschiede zwischen der Mass Media Logic und der Network Media Logic gebe. Den Autor_innen folgend zeichnet die Network Media Logic vor allem die kostengünstige Produktion und Beschaffung von Inhalten entsprechend den Bedürfnissen der Nutzer_innen aus; ferner würden Nutzende selbst zunehmend als Intermediäre agieren und populäre Inhalte in ihren Netzwerken verbreiten, die vor allem aus Gleichgesinnten bestünden. Zudem heben Klinger und Svensson in ihren Ausführungen die Wichtigkeit von Blogger_innen und Lai_innen im politischen Kontext hervor, da diese als Intermediäre für die virale Distribution von Inhalten agieren würden. Bei allen Autor_innen handelt es sich in Abgrenzung zu klassischen Medienlogiken (z. B. Personalisierung, Skandalisierung) um neue Kommunikationslogiken, die von Digitalplattformen und potentiell auch im professionellen Journalismus umgesetzt werden, im Sinne von Anpassungstendenzen. Plattformlogiken nach Eisenegger (2021) zeichnen sich nicht nur durch ihre algorithmische Kodierung aus, sondern auch durch Plattform-Affordanzen, also die Strukturmerkmale von Plattformen (z. B. Design, Funktionen wie Likes und Shares) und den Plattform-Gebrauch, im Sinne des Handelns der Nutzer_innen, das durch Algorithmen observiert und datafiziert wird. Gesamthaft skizziert Eisenegger (2021, S. 35) Öffentlichkeit im digitalen Zeitalter als plattformisierte Longtail-Öffentlichkeit, geprägt durch eine «Deinstitutionalisierung journalistischer Informationsmedien» und eine «Institutionalisierung neuer Plattformlogiken».

Die hier skizzierte Beschreibung des digitalen Strukturwandels der Öffentlichkeit verdeutlicht, dass sich vor allem aufgrund der Etablierung von Digitalplattformen und damit einhergehenden neuen Logiken die öffentliche Kommunikation gewandelt hat. Stellenweise zeigte sich bereits, dass diese Transformation vor allem Herausforderungen für die öffentliche Kommunikation respektive den öffentlichen Diskurs impliziert. Im folgenden Kapitel werden damit einhergehende Problematiken kurz resümiert. 


\section{II.3.3 Herausforderungen digitaler Öffentlichkeit(en)}

Bevor im abschliessenden Zwischenfazit eine kritische Betrachtung neuer Öffentlichkeitskonzepte im Vergleich zu klassischen Theorien erfolgt, soll an dieser Stelle noch ein kurzer Überblick über aktuelle Phänomene gegeben werden, die die digitale Öffentlichkeit potentiell prägen. In diesem Zusammenhang soll vor allem auf die bereits im vorigen Kapitel angedeutete Gefahr einer Publikumsfragmentierung eingegangen werden, die eng mit der Herausbildung individueller $\mathrm{Me}$ dienrepertoires und den - sehr wohl strittigen - Phänomenen der «Echokammern» und «Filterblasen» in Zusammenhang steht. Dabei handelt es sich lediglich um einen Ausschnitt von - dem digitalen Öffentlichkeitswandel - geschuldeten Phänomenen, die aufgrund der Thematik der vorliegenden Arbeit reflektiert werden müssen.

So beschreibt beispielsweise Stark (2013) das Phänomen der Publikumsfragmentierung als Folge der Veränderung der Medienlandschaft im Internetzeitalter. Aufgrund einer Ausdifferenzierung von Nachrichtenangeboten, beispielsweise über Plattformen als neue Verbreitungskanäle, und einer damit einhergehenden stärker interessen- und bedürfnisorientierten Nutzung dieser Angebote wird eine «Fragmentierung des Medienpublikums» (oder auch: «Differenzierung», «Segmentierung», «Polarisierung») im Sinne einer Desintegration des Publikums angenommen (Hasebrink, 2008; Prior, 2005, 2007; Stark, 2013, S. 199f.; Stroud, 2010, 2011). Eine «Zersplitterung des Publikums» führe dazu, dass das Publikum «weniger gemeinsame medial vermittelte Erfahrungen mache, was wiederum negative gesellschaftliche Konsequenzen habe», im Sinne mangelnder gesellschaftlicher Integration (Kleinen-von Königslöw, 2016, S. 254). Das Konzept der Publikumsfragmentierung hat eine lange Tradition in der Medienwissenschaft und wurde nicht erst im Zuge des digitalen Wandels aufgegriffen; so spricht beispielsweise bereits Habermas (2018 [1962], S. 57) von einem potentiellen «Zerfall der Öffentlichkeit» und dessen Gefahren für die Demokratie. Spätestens durch die Einführung des Fernsehens wurde der Begriff der Fragmentierung als Ausdifferenzierung von Teilpublika diskutiert (Hasebrink \& Rössler, 1999; Holtz-Bacha, 1997; Kleinen-von Königslöw, 2016). Aufgrund einer zunehmenden «Plattformisierung» (Eisenegger, 2021; Helmond, 2015) und entsprechender Algorithmisierung in Form von personifizierten und über Codes gestreuten Medienangeboten kann allerdings die These aufgestellt werden, dass sich die Gefahr einer zunehmenden Fragmentierung weiter verstärkt. Aktuelle Befunde dazu sind allerdings widersprüchlich und prognostizieren teilweise eine zunehmende Fragmentierung oder auch eine dennoch stattfindende überlappende Themenwahrnehmung, und hängen - so Stark (2013) - deutlich von der jeweiligen Analyseebene ab, wobei zwischen medien-, publikums- und nutzerzentrierten Ansätzen unterschieden werden kann. Medienzentrierte Ansätze fokussieren vor allem die Aufmerk- 
samkeitsverteilung im Sinne einer Marktverteilung von Medienangeboten, publikumszentrierte Ansätze untersuchen die Publikumszusammensetzungen für Mediengattungen wie auch die Repertoires von Publikumssegmenten und nutzerzentrierte Ansätze analysieren individuelle Medienrepertoires als persönliche Nachrichtenbündel. Eng verbunden mit dem Konzept der Medienrepertoires als die Gesamtheit von Medien, die Individuen regelmässig nutzen (Hasebrink \& Popp, 2006), ist jenes der Newsrepertoires, als «die Gesamtheit derjenigen (News-) Medien, die eine Person nutzt, um sich über aktuelle Geschehnisse zu informieren» (Schneider \& Eisenegger, 2019, S. 27), im Sinne von individuellen Nutzer_innenpräferenzen (vgl. hierzu auch Edgerly, 2015; Swart, Peters \& Broersma, 2017). Schneider und Eisenegger (2019) erkennen dabei vor allem im Zeitalter der Digitalisierung und der Etablierung von Social-Media-Plattformen die Gefahr, dass die Nachrichtennutzung zunehmend mit interpersoneller Kommunikation, sozialer Kontaktpflege und Unterhaltung in Konkurrenz steht. Zudem erweist sich die Mediennutzung in digitalen Gesellschaften als zunehmend emergent, wie bereits im vorigen Kapitel ausgeführt wurde (Eisenegger, 2021; Schwaiger, 2019).

Durch die Etablierung von Digitalplattformen und die personalisierte Selektion von Online-Beiträgen über Algorithmen werden in diesem Zusammenhang Phänomene der «Filterblasen» und «Echokammernbildung» diskutiert, die eng miteinander verzahnt sind. Pariser (2011) prägte massgeblich den Begriff der «Filter Bubbles» und beschreibt damit die Problematik, dass Nutzende von Social Media hauptsächlich jene Beiträge rezipieren, die ihren persönlichen Interessen und Nutzer_innengewohnheiten entsprechen, da diese algorithmisch selektiert werden. Aus demokratiepolitischer Sicht würde dies eine Desintegration der Gesellschaft bedeuten. Eine Folge dieser gefilterten Beitragsselektion wäre das Entstehen von Echokammern, in denen ein Diskurs hauptsächlich unter Gleichgesinnten stattfindet und Beiträge rezipiert werden, die die eigene Meinung und persönliche Ansichten widerspiegeln (Flaxman, Goel \& Rao, 2016). Dies würde in einer weiteren Polarisierung respektive Fragmentierung der Gesellschaft resultieren. Aktuelle Forschungen revidieren allerdings die tatsächlichen Gefahren der beiden Phänomene (z. B. Dubois \& Blank, 2018; Haim, Graefe \& Brosius, 2018; Zuiderveen Borgesius et al., 2016). Auch in diesen Fällen scheint der jeweilige methodische Zugang zu teils unterschiedlichen empirischen Resultaten zu führen. Aus theoretischer Sicht - in Abgrenzung dazu - sieht beispielsweise Pörksen (2018) eine Gefahr zunehmender Polarisierung, selbst - oder gerade dann - wenn unterschiedlich gesinnte Personen oder Gruppen via Social Media gegenseitig Beiträge kommentieren und diskutieren, dies aber nicht auf der Aushandlung des besseren Arguments beruht. Der Autor bezeichnet dieses Phänomen als «Filter-Clash» und beschreibt damit den konflikthaften Diskurs von Parallelöffentlichkeiten, der die Bestätigung eigener Ansichten noch weiter evoziere. 


\section{II.3.4 Zwischenfazit: «Digitale» vs. «klassische» Öffentlichkeitskonzepte}

In den letzten Kapiteln wurden unterschiedliche theoretische Ansätze diskutiert, die sich mit dem Begriff der «Öffentlichkeit» auseinandersetzen. Dabei wurde zunächst versucht, einen Überblick über klassische Öffentlichkeitskonzepte zu geben: Einerseits normative Ansätze unter Bezug auf Habermas (2018 [1962]), systemtheoretische Ansätze mit Verweis auf Luhmann (2017 [1995]) sowie das analytische Modell nach Gerhards und Neidhardt (1991). Deren wesentlichen Merkmale wurden bereits diskutiert, von Interesse ist aber vor allem deren Vergleich mit aktuellen Öffentlichkeitskonzepten, wie dem der Netzwerköffentlichkeit (z. B. Benkler, 2006; Castells, 2010 [1996]; Friedland et al., 2014) oder der Plattformöffentlichkeit (z. B. Eisenegger, 2021; Helmond, 2015). Fraglich ist, ob es neuer, digitaler Öffentlichkeitskonzepte bedarf bzw. welche Elemente aus klassischen Konzepten gegebenenfalls adaptiert oder übernommen werden können.

Die Annahme eines digitalen Strukturwandels der Öffentlichkeit nimmt schon ob seiner Bezeichnung Bezug auf Habermas' (2018 [1962]) Theorie. Habermas beschreibt im «Strukturwandel der Öffentlichkeit» wesentliche Elemente, die eine Transformation der öffentlichen Kommunikation zur Folge hatten. Eine Erweiterung des Konzepts für das digitale Zeitalter ist folglich naheliegend. Obwohl der normative Ansatz von Habermas aus unterschiedlichen Gründen kritisiert werden kann (vgl. hierzu auch Kapitel III.1), ist eine idealtypische Betrachtung, wie öffentliche Kommunikation funktionieren sollte, auch in digitalen Öffentlichkeiten essentiell. Im digitalen Raum stellt sich allerdings die Frage, ob dieser Idealtyp überhaupt noch umsetzbar und z. B. ein argumentbasierter Diskurs auf Plattformen empirisch gegeben ist, oder aber Polarisierungs- respektive Fragmentierungstendenzen (Holtz-Bacha, 1997) das normative Konzept ins Wanken bringen. Aus einer systemtheoretischen Perspektive lässt sich vor allem die Logik der Codes ableiten. So zeigen Autor_innen aktuell, dass vor allem Plattformen von sogenannten Social Media Logics (van Dijck \& Poell, 2013), Plattformlogiken (Eisenegger, 2021) oder Network Media Logics (Klinger \& Svensson, 2015) geprägt sind. Diese vor allem auf Algorithmen zurückzuführenden Logiken spiegeln sich in vielen Öffentlichkeitskonzepten seit der Jahrtausendwende wider, so beispielsweise auch in Castells' Ausführungen zur Netzwerkgesellschaft (2010 [1996]). Da moderne, digitale Öffentlichkeiten fluide sind, ist weiter eine Trennung in unterschiedliche Öffentlichkeitsebenen, wie von Gerhards und Neidhardt (1991) vorgeschlagen, kaum mehr denkbar. Digitale Öffentlichkeiten sind vor allem dadurch geprägt, dass die Barrieren, öffentlich zu kommunizieren, gesunken sind; potentiell kann jede_r zum/zur Kommunikator_in werden, der professionelle Journalismus ist nicht mehr Gatekeeper der Informationsverbreitung. Unabhängig davon, wie die moderne, digitale Öffentlichkeit bezeichnet wird, scheint es plausibel, dass vor allem strukturelle Veränderungen, die durch die Digitalisierung 
angetrieben wurden, die öffentliche Kommunikation massgeblich beeinflusst haben. $\mathrm{Zu}$ betonen ist diesbezüglich die Etablierung von Digitalplattformen, die mit eigenen Logiken operieren und somit die öffentliche Kommunikation massgeblich steuern (Eisenegger, 2021; Helmond, 2015).

Bezogen auf die Annahme einer Plattformöffentlichkeit stellt sich die Frage, ob sie das aktuelle oder auch künftige Öffentlichkeitsmodell moderner, digitaler Gesellschaften abbildet, oder als Art Teilöffentlichkeit zu betrachten ist. Es ist einerseits davon auszugehen, dass das hybride Mediensystem nicht ausschliesslich durch Plattformen geprägt ist: Wenn auch mit sinkenden Nutzer_innenzahlen, existieren weiterhin traditionelle Medienangebote wie die Presse, das Radio oder das Fernsehen. Daher lässt sich annehmen, dass auch traditionelle Medienkonzerne (vor allem unter Bezugnahme auf eine neoinstutionalistische Perspektive) Plattformlogiken adaptieren und der gesellschaftlichen Erwartungshaltung einer Präsenz auf Digitalplattformen gerecht werden (Eisenegger, 2021). Bestimmte User_innengruppen - und darunter vor allem die Zielgruppe der unter 30-Jährigen - konstruieren sich zudem ihr Bild der Gesellschaft nachweisbar mehr und mehr über Social-Media-Plattformen. ${ }^{4}$ Insofern ist die Theorie einer plattformisierten Öffentlichkeit durchaus ergebnisträchtig. Noch zu wenig hinterfragt ist dabei die Publikumsperspektive, vor allem, wenn an die ursprünglichen von Habermas (2018 [1962]) abgeleiteten Kriterien von Öffentlichkeit gedacht wird: Ist in der Plattformöffentlichkeit tatsächlich ein freier Zugang zum Diskurs unabhängig von persönlichen oder statusbasierten Eigenschaften möglich und denkbar? Oder schliessen soziale Kriterien bestimmte Personengruppen vom Diskurs aus, im Sinne einer sozialen Ungleichheit (Stichwort: Digital Divide (z. B. Bach, Wolfson \& Crowell, 2018)? Und gibt es überhaupt die Plattformöffentlichkeit oder handelt es sich vielmehr um plattformabhängige Teilöffentlichkeiten, wie beispielsweise die Twittersphäre oder Instagram-Öffentlichkeit, die unterschiedliche Publika implizieren? In diesem Zusammenhang (auch empirisch) noch zu wenig beachtet ist weiter die Ausformierung des «Longtails»: Sind reichweitenschwache Akteur_innen noch Teil der Öffentlichkeit respektive öffentlichen Meinung? Handelt es sich allenfalls um Gegenöffentlichkeiten, die sich innerhalb des Longtails um Diskurse strukturieren und neu konstruieren? Und können diese durch eine Vernetzung untereinander - als Adaption der Plattformlogik (Eisenegger, 2021) - den vorderen Teil der Öffentlichkeit erreichen und gesamthaft Reichweiten erzielen?

Den digitalen Strukturwandel theoretisch wie auch empirisch $\mathrm{zu}$ fassen, stellt ob seiner Mehrdimensionalität eine Herausforderung dar. Mehrdimensio-

4 So sind es beispielsweise unter den 18- bis 24-jährigen Schweizer_innen im Jahr $202034 \%$, die hauptsächlich Nachrichten über Social Media konsumieren. Zum Vergleich: Im Vorjahr 2019 waren es noch $25 \%$ (Schwaiger et al., 2020). 
nal ist dieser deshalb, da sich sowohl auf struktureller Ebene (z. B. Etablierung von Plattformen, Veränderung des Mediensystems) als auch inhaltlicher Ebene (z. B. Plattformlogiken) ein Wandel abzeichnet. Dieser äussert sich überdies erstens in der Zeitdimension, wonach sich die Digitalisierung nicht nur in einer «Always-On-Gesellschaft» (Koningsbruggen, Hartmann \& Du, 2018) niederschlägt, sondern auch publizistische Tätigkeiten rund um die Uhr geschehen und erwartet werden. Die traditionelle Tageszeitung ist dieser Logik folgend zum Zeitpunkt der Veröffentlichung häufig bereits überholt, weshalb auf Online-Pendants zurückgegriffen wird. Zweitens vollzieht sich der Wandel auf der Sachdimension. Themenagenden und Inhalte werden durch den digitalen Wandel nicht nur mitbestimmt, sondern basieren neben «traditionellen» Medienlogiken allenfalls auch auf neuen Social-Media- respektive Plattformlogiken (Eisenegger, 2021; van Dijck \& Poell, 2013). Drittens ist die Sozialdimension zu nennen: Die digitale Transformation ermöglicht es unterschiedlichen Akteur_innen, Öffentlichkeit herzustellen, die vormals nicht in diesem Ausmass die Möglichkeit hatten, Resonanz bei einem grossen Publikum zu erreichen. $\mathrm{Zu}$ relativieren ist diesbezüglich gleichwohl die Neuartigkeit dieser Akteur_innen. Mit Blick auf die bürgerliche Öffentlichkeit (noch vor dem ersten Strukturwandel und der Etablierung der Zeitung als Massenmedium) war es idealtypisch erforderlich, dass ein_e jede_r am Diskurs teilhaben, also öffentlich kommunizieren konnte (Habermas, 2018 [1962]). Auch Gerhards' und Neidhardts (1991) Drei-Ebenen-Modell weist mindestens auf der Encounter-, allenfalls auch auf der Versammlungsebene, auf die Diversität der zu Wort kommenden Individuen hin, beispielsweise in Form von Protestbewegungen innerhalb der Versammlungsöffentlichkeit. So war es potentiell seit jeher jedem bzw. jeder möglich, Öffentlichkeit herzustellen, wenn auch mit kleinerem Publikum ${ }^{5}$. Dennoch bleibt unbestreitbar, dass die digitale Transformation die Möglichkeiten, öffentlich zu kommunizieren, potenziert hat. Gerhards' und Neidhardts (1991) Begriffsarchitektur folgend kann einerseits von einer Ausweitung der Encounter-Ebene durch das Internet gesprochen werden und andererseits von einer damit einhergehenden zunehmenden Vermischung der drei Ebenen, die in digitalen Öffentlichkeiten wohl besser als eine Art Kontinuum zu betrachten sind. An diesem Punkt erscheint das Bild einer Longtail-Öffentlichkeit griffig, wie sie von Neuberger (2009) und Eisenegger (2017) aufgegriffen wurde. Diesbezüglich gilt es festzuhalten, dass durch das Internet und die Etablierung von Digitalplattformen die Anzahl an (öffentlichen) Kommunikator_innen angestiegen ist und diese vor allem öffentlich sichtbar wurden. Zudem sind die Barrieren für Lai_innen gesunken, ein grösseres Publikum und Resonanz zu erreichen - man denke beispielsweise an Influencer_innen, die ausnahmsweise den vorderen Teil des Longtails

5 Inwiefern dies unterprivilegierten Gruppen (z. B. politischen Randgruppen oder auch Frauen) tatsächlich immer möglich war, ist in Frage zu stellen - vgl. hierzu Fraser (1996), Kapitel III.1. 
erreichen und Deutungsmacht erlangen. Aber insbesondere im journalistischen Bereich ist die Annahme eines Strukturwandels treffend. Hervorzuheben ist dabei die verminderte Gatekeeping-Funktion des professionellen Journalismus, der nicht mehr alleine für die Selektion und Publikation von Nachrichtenbeiträgen verantwortlich ist (Wallace, 2017). Theoretisch wird davon ausgegangen, dass im journalistischen Bereich beispielsweise Laienkommunikator_innen, aber auch Nachrichtenmedien abseits des professionellen Informationsjournalismus - sogenannte alternative Nachrichtenmedien - von de facto kaum vorhandenen Publikationsbarrieren Gebrauch machen. Dies kann einerseits aus einer demokratischen Perspektive als positiv erachtet werden, da die gesellschaftliche Kritik und Kontrolle gegenüber (medialen, politischen oder anderen) Eliten jeder bzw. jedem potentiell möglich sein sollte. In der Sozialforschung wird ebendieser Punkt allerdings kritisch betrachtet: Die Möglichkeit, ohne Zugangsbarrieren öffentlich aufzutreten und Inhalte zu verbreiten, birgt schliesslich die Gefahr, dass die Einhaltung journalistischer Qualitätsstandards nicht mehr gewährleistet ist. Diese ausgehende Gefahr potenziert sich, wenn für die Rezipierenden nicht mehr eindeutig unterscheidbar ist, ob es sich um professionelle oder laienhafte Informationen handelt, wie es beispielsweise auf Social-Media-Plattformen der Fall sein kann, da hier im persönlichen Newsfeed unterschiedliche Medienmarken gebündelt und konkrete Marken nicht mehr gezielt angesteuert werden müssen. Auf Digitalplattformen ist es durch diese Medienemergenz (Eisenegger, 2021) schliesslich auch möglich, dass einzelne - gegebenenfalls auch von Lai_innen produzierte - Beiträge Resonanz erzielen, nämlich in Form von Likes, Retweets o. Ä. Diese Social-Media- oder Plattformlogiken können dabei als Treiber für Deutungsmacht in digitalen Öffentlichkeiten betrachtet werden. Neben der Verleihung von Likes etc. spielt hier auch die Vernetzung als Social-Media-Logik eine wesentliche Rolle (Klinger \& Svensson, 2015). In diesem Zusammenhang gehe ich davon aus, dass einzelne Akteur_innen am «langen Schweif» des Longtails vor allem dadurch Resonanz respektive Definitionsmacht erlangen, da sie sich untereinander vernetzen. Dabei scheinen sich vor allem homophile, gleichgesinnte Gruppen um bestimmte Diskurse zu strukturieren und als Teilöffentlichkeiten zu formieren. Durch die Vernetzung untereinander, beispielsweise durch das gegenseitige Folgen oder Abonnieren auf den Plattformen, oder durch das gegenseitige Teilen von Beiträgen, kann die jeweilige Popularität einzelner Akteur_innen erhöht werden, da deren Inhalte noch weiter gestreut werden. Dadurch wird es sowohl einzelnen Akteur_innen möglich, den vorderen Teil des Longtails zu erreichen, aber vor allem auch jenen herausgebildeten Teilöffentlichkeiten als Ganzes. Jene Teilöffentlichkeiten können dann - je nach Diskurs - die Rolle von Gegenöffentlichkeiten einnehmen. Gegenöffentlichkeit deshalb, weil sie eine oppositionelle Haltung gegenüber der öffentlichen Meinung medialer und politischer Eliten einnehmen. So zeigt beispielsweise Lewis (2018) in ihrer Forschung zum 
«Alternative Influence Network», wie sich bestimmte politische Influencer_innen auf YouTube miteinander vernetzen und so Publika mit radikalen politischen Positionen (z. B. Far-Right-Ideologien) ansprechen.

Ich gehe weiter davon aus, dass vor allem in modernen, digitalen Öffentlichkeiten dem Gegenöffentlichkeitskonzept eine besondere Bedeutung zugeschrieben werden muss. Die digitale Transformation ermöglicht es schliesslich, dass sich unterschiedliche Akteur_innen auf simple Art und Weise miteinander vernetzen können - und das orts- und zeitunabhängig. Durch ihre Organisation und Strukturierung über Digitalplattformen können sie ein grösseres Publikum ansprechen und mobilisieren, wie es wohl in modernen Gesellschaften vor der Digitalisierung und Plattformisierung kaum möglich gewesen wäre. Diese These wird im folgenden Kapitel erneut aufgegriffen. Der Begriff «Gegenöffentlichkeit» wird hierfür theoretisch eingeordnet und für das Phänomen «alternative Nachrichtenmedien» spezifiziert. 
